\title{
Discovery of three optical open clusters in the Galaxy
}

\author{
E. Bica ${ }^{1}$, C. Bonatto ${ }^{1}$, and C. M. Dutra ${ }^{2}$ \\ 1 Universidade Federal do Rio Grande do Sul, Instituto de Física, CP 15051, Porto Alegre 91501-970, RS, Brazil \\ e-mail: charles@if.ufrgs.br \\ 2 Universidade Estadual do Rio Grande do Sul, Unidade São Borja, Rua Bompland 512, São Borja 97670-000, RS, Brazil
}

Received 14 January 2004 / Accepted 31 March 2004

\begin{abstract}
We report the discovery of three optical open clusters in the Milky Way. Two clusters are in Scutum (Cluster 1 at $\ell=18.44^{\circ}$ and $b=-0.42^{\circ}$, and Cluster 2 at $\ell=19.60^{\circ}$ and $b=-1.02^{\circ}$ ), thus projected not far from the Galactic center direction, and the other is in Canis Major (Cluster 3 at $\ell=235.61^{\circ}$ and $b=-4.10^{\circ}$ ), near the anti-center direction. Cluster 3 is less populous than Clusters 1 and 2, but presents evidence of being a physical system. The objects were found optically by inspecting maps obtained from the Guide Star Catalogue and images from the Digitized Sky Survey. No previous identification of a cluster has been reported in any of these areas so far. The analysis was carried out with 2MASS photometry in $J$ and $H$. For Cluster 1 we derive an age of $t=25 \pm 5 \mathrm{Myr}$, a reddening $E(B-V)=2.18 \pm 0.03$ and a distance from the Sun $d_{\odot}=1.64 \pm$ $0.19 \mathrm{kpc}$; for Cluster 2, $t=500 \pm 100 \mathrm{Myr}, E(B-V)=0.91 \pm 0.03$ and $d_{\odot}=2.19 \pm 0.21 \mathrm{kpc}$; finally for Cluster 3, $t=32-100 \mathrm{Myr}, E(B-V)=0.94 \pm 0.03$ and $d_{\odot}=3.93 \pm 0.35 \mathrm{kpc}$. Luminosity and mass functions are derived for Clusters 1 and 2 which, in turn, allow us to estimate their observed masses as $147 M_{\odot}$ and $89 M_{\odot}$, respectively. Estimated total masses, by extrapolating the mass functions to $0.08 M_{\odot}$, amount to $382 M_{\odot}$ and $614 M_{\odot}$ for the two clusters. Cluster 3 has an observed mass of $\sim 55 M_{\odot}$. The present results indicate that further searches in the optical might still reveal new open clusters, especially in the infrared bands.
\end{abstract}

Key words. Galaxy: open clusters and associations: general - Galaxy: structure

\section{Introduction}

The discovery of new physical stellar systems in the Galaxy is essential for completing their census. This, along with the determination of properties such as distance, age, mass and dynamical state, and spatial distribution and evolutionary processes in the disk (e.g., Janes \& Adler 1982; Twarog et al. 1997) will give a better statistical insight in their formation, evolution and eventual disruption. Open clusters span a wide range of ages from very young to intermediate age, and their ages and distances can be determined by fitting isochrones to their colour-magnitude diagrams (CMDs), with a precision depending on the depth of the photometry and on the field contamination. These objects are formed along the Galactic plane where there is an abundance of gas and dust and their orbits become perturbed by the cumulative effect of passages near interstellar clouds. Due to their spatial location, young open clusters can be considered tracers of the Galaxy's spiral structure (Chen et al. 2003). Thus, the discovery and characterization of additional open clusters represent a step further in the understanding of Galaxy structure and Galaxy formation processes.

In the recent decades some new open clusters have been discovered in the optical domain, e.g. Pfleiderer et al. (1977), Turner et al. (1986) and Saurer et al. (1994). Recently, two optical open clusters in Cygnus OB2 were found and their properties determined (Bica et al. 2003). In the infrared, the number of new embedded clusters is striking, mostly due to the recent release of the Two Micron All Sky Survey (hereafter 2MASS, Skrutskie et al. 1997) catalogue and atlas. We point out the recent discovery of 346 embedded clusters and candidates in the spiral arms of the Galaxy (Dutra et al. 2003a; Bica et al. 2003).

In the present study we report finding three new optical open clusters. Their properties are derived by means of 2MASS photometry, since these optical objects show up clearly in the near-infrared. In addition, the uniform and essentially complete sky coverage provided by 2MASS allows us to properly take into account background regions with suitable star count statistics, which is fundamental for the correct identification and characterization of the stellar content of clusters.

In Sect. 2 we present the new open clusters and show the optical images of the areas where the clusters have been found. In Sect. 3 we obtain the 2MASS photometry and introduce the $J \times(J-H)$ CMDs. In Sect. 4 we discuss the radial density distribution of stars and derive structural parameters for the clusters. In Sect. 5 we fit isochrones to the near-infrared CMDs and derive cluster parameters. In Sect. 6 we derive the luminosity and mass functions (hereafter LF and MF) and estimate the stellar masses of each cluster. Concluding remarks are given in Sect. 7. 


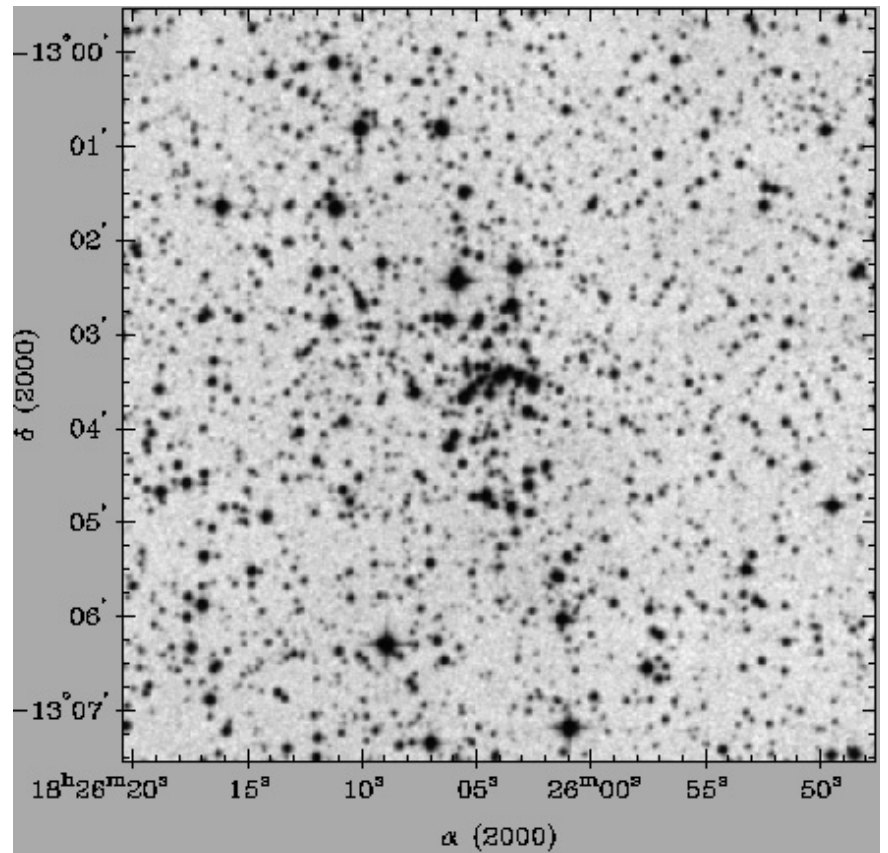

Fig. 1. $8^{\prime} \times 8^{\prime}$ XDSS $R$ image of Cluster 1 .

\section{The three new open clusters}

The clusters have been found during systematic inspections of the Milky Way with maps generated by means of the Guide Star Catalogue, Sky Survey Charts and detailed charts of candidate regions with Digitized Sky Survey (DSS and XDSS) fields. Equatorial and Galactic coordinates, and angular diameters are given in Table 1.

Clusters 1 and 2 are in Scutum, thus projected not far from the direction of the Galactic center, while Cluster 3 is in Canis Major, not very far from the anti-center. None of these objects has been listed in previous catalogues (Alter et al. 1970; Lyngå 1987; Dias et al. 2002).

We show in Figs. 1-3 the three open clusters in optical bands. Clusters 1 and 2 are projected against rich fields, as expected from their Galactic coordinates. Considering the stellar distributions, the three objects stand out from the background areas. Open Cluster 1 is elongated in the North-South direction and rather concentrated to the center (Fig. 1). Although Cluster 2 is looser than Cluster 1 it still stands out from the rich background (Fig. 2). Finally, Cluster 3, although poorer in stars, presents a central concentration.

Cluster 1 is projected approximately $16^{\prime}$ to the North-East of the H II region Sh 2-53 (Sharpless 1959). Cluster 2 lies approximately $13^{\prime}$ West of the open cluster Ruprecht 141 (Alter et al. 1970) for which no parameters are available, according to the open cluster database WEBDA (Mermilliod 1996 http: //obswww . unige. ch/webda). Finally, Cluster 3 is projected approximately $17^{\prime}$ Southeast of the young open cluster NGC 2367 - with age $\approx 5$ Myr (Vogt \& Moffat 1972) and 5' South of the HII region RCW 14 (Rodgers et al. 1960). RCW 14 appears to be the nucleus of the larger nebula Brand 16 (Brand et al. 1986). The projected environments of Clusters 1 and 3 thus contain young objects.

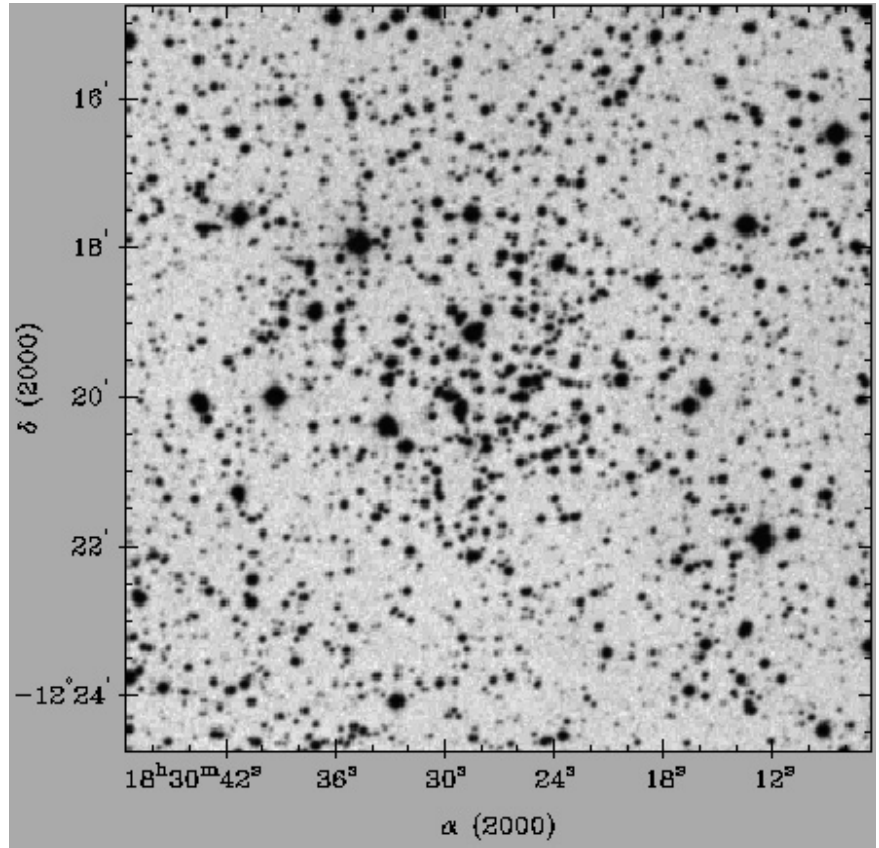

Fig. 2. $10^{\prime} \times 10^{\prime}$ DSS $B$ image of Cluster 2 .

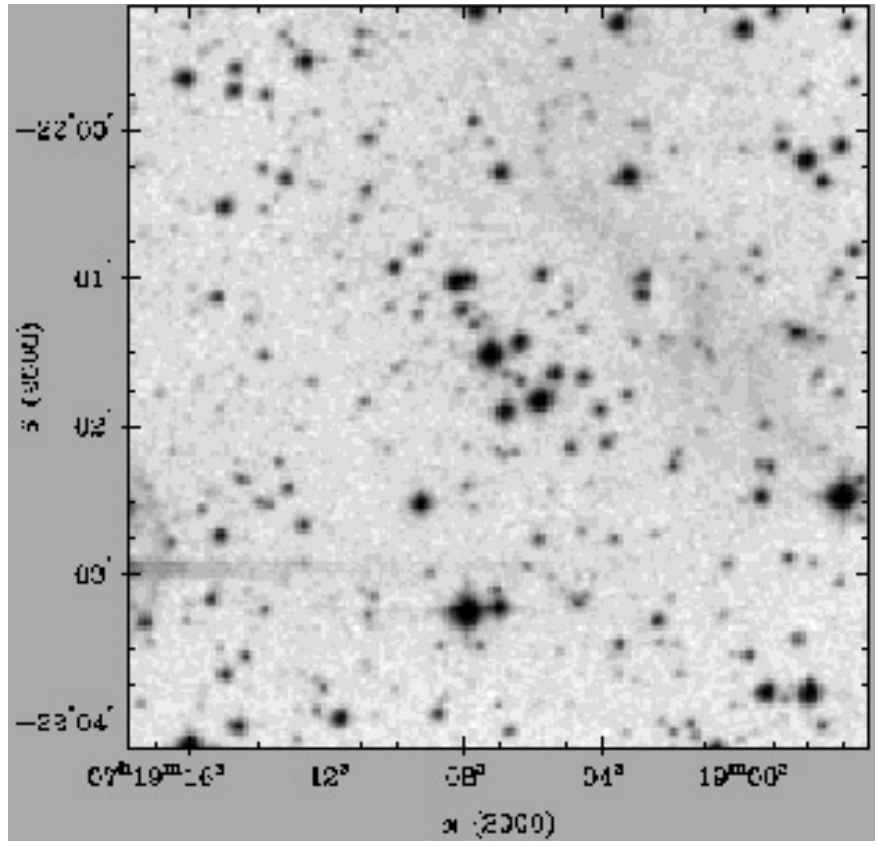

Fig. 3. $5^{\prime} \times 5^{\prime}$ XDSS $R$ image of Cluster 3 .

\section{The 2MASS photometry}

$J$ and $H$ photometry has been obtained from the 2MASS All Sky data release, available at http ://www . ipac . caltech . edu/2mass/releases/allsky/. 2MASS photometric errors typically attain 0.10 mag at $J \approx 16.2$ and $H \approx 15.0$, see e.g. Soares \& Bica (2002). For each cluster we made circular extractions centered on the coordinates given in Table 1 with the extraction radius $\left(R_{\mathrm{CMD}}\right)$ listed in Col. 7 . We decided on an extraction area smaller than the optical one to minimize background 
Table 1. Observational parameters for the new clusters.

\begin{tabular}{cccccccc}
\hline \hline Object & $\alpha(2000)$ & $\delta(2000)$ & $\ell$ & $b$ & Diameter & $R_{\mathrm{CMD}}$ & $E(B-V)_{\text {FIR }}$ \\
\hline Cluster 1 & $18^{\mathrm{h}} 26^{\mathrm{m}} 04^{\mathrm{s}}$ & $-13^{\circ} 03^{\prime} 32^{\prime \prime}$ & $18.44^{\circ}$ & $-0.42^{\circ}$ & $3.5^{\prime} \times 2.5^{\prime}$ & $1.5^{\prime}$ & 15.77 \\
Cluster 2 & $18^{\mathrm{h}} 30^{\mathrm{m}} 30^{\mathrm{s}}$ & $-12^{\circ} 18^{\prime} 59^{\prime \prime}$ & $19.60^{\circ}$ & $-1.02^{\circ}$ & $6.0^{\prime} \times 6.0^{\prime}$ & $2.0^{\prime}$ & 5.63 \\
Cluster 3 & $07^{\mathrm{h}} 19^{\mathrm{m}} 07^{\mathrm{s}}$ & $-22^{\circ} 01^{\prime} 40^{\prime \prime}$ & $235.61^{\circ}$ & $-4.10^{\circ}$ & $2.5^{\prime} \times 2.5^{\prime}$ & $1.1^{\prime}$ & 1.66 \\
\hline
\end{tabular}

Table notes. Column 6 gives the optical diameters; Col. 7 gives the extraction radius used in the CMD analyses; $E(B-V)_{\text {FIR }}$ is Schlegel et al.'s (1998) reddening values, derived from the far-infrared dust emission.

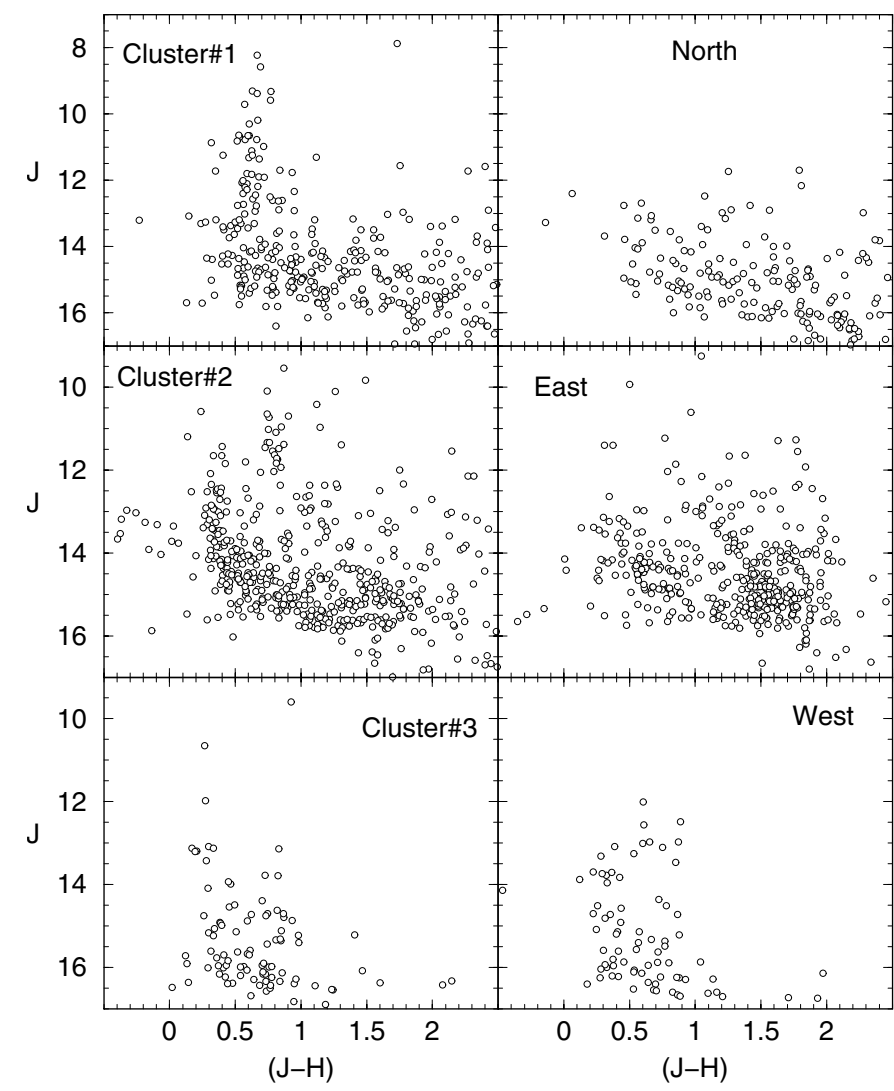

Fig. 4. $J \times(J-H)$ CMDs for the new clusters (left panels); representative offset fields are also shown (right panels).

contamination and increase the membership probability of the stars sampled. Comparison fields have been extracted inside circular areas with the same radii as those used for the clusters in four positions North, South, East and West of each cluster, with center to center distances corresponding to $3 \times R_{\mathrm{CMD}}$. The extractions were performed using the VizieR tool at http://vizier.u-strasbg.fr/ viz-bin/VizieR?-source=2MASS. In Fig. 4 we show the $J \times(J-H)$ CMDs for each cluster (left panels) along with those of a representative offset field (right panels).

In the fields of Clusters 1 and 2 star colours reach as much as $(J-H)=3$, beyond the limits of Fig. 4, while for the Cluster 3 area all stars are included in the figure. These very red colours, which correspond to high reddening values, are caused by accumulation of dust in the line of sight, since we are dealing with very low Galactic latitudes for Clusters 1 and 2 (Table 1). This is confirmed by Schlegel et al.'s (1998) reddening values derived from dust emission in the far-infrared (FIR), given in the last column of Table 1. Dutra \& Bica (2002) studied low-latitude directions using star clusters as probes for reddening in the foreground and background of the clusters. More recently, Dutra et al. (2003b) found evidence that the calibration should be corrected, viz. that Schlegel et al.'s values should be multiplied by 0.75 , at least for low Galactic latitudes towards the Galactic center (down to $|b|=4^{\circ}$ ). Anyhow, the $E(B-V)_{\mathrm{FIR}}$ values in the directions of Clusters 1 and 2 would still be very high.

Cluster 1 presents a prominent main sequence (MS) compared to the corresponding offset field (Fig. 4), which is indicative of a young age. Cluster 2 exhibits a MS and giants, indicating older ages. Cluster 3 presents evidence of a young MS.

\section{Cluster structure}

For the specific purpose of better accessing the overall cluster structure, we made additional star extractions reaching as far as the optical diameter of each cluster, according to the values in Table 1. With the stars obtained in these new extraction areas, we built the star density radial distributions, defined as the number of stars per area, in and around the clusters, which are shown in Fig. 5.

Before counting stars, we applied a cutoff $(J<15.5)$ to Clusters 1 and 2 and their corresponding offset fields to avoid undersampling, i.e. to avoid spatial variations in the number of faint stars which are numerous, affected by large errors, and may include spurious detections in the area of the clusters. Colour filters have also been applied to both Clusters 1 and 2 and offset fields, to account for the contamination of the Galaxy - only stars with colours in the range $0.0 \leq(J-H) \leq$ 1.1 have been considered. This procedure has been applied in the analysis of the open cluster M 67 (Bonatto \& Bica 2003). Because of the relatively small number of stars in the area of Cluster 3, no cutoff was applied. The radial distribution was determined by counting stars inside concentric annuli with a step of $0.25^{\prime}$ in radius up to the new extraction limits. The background contribution, shown in Fig. 5 as shaded rectangles, corresponds to the average number of stars included in the four offset fields.

Cluster 1 has a slight deficiency of stars near the center compared to the neighbouring annulus, but beyond $R=0.25^{\prime}$ it presents a well-defined and rather smooth profile (top panel) with star counts well above the background, considering the Poisson errors. The central deficiency might be partly accounted for by faint star images blended with the several bright ones near the object center. According to the radial distribution of stars, Cluster 1 extends beyond the CMD extraction radius, 


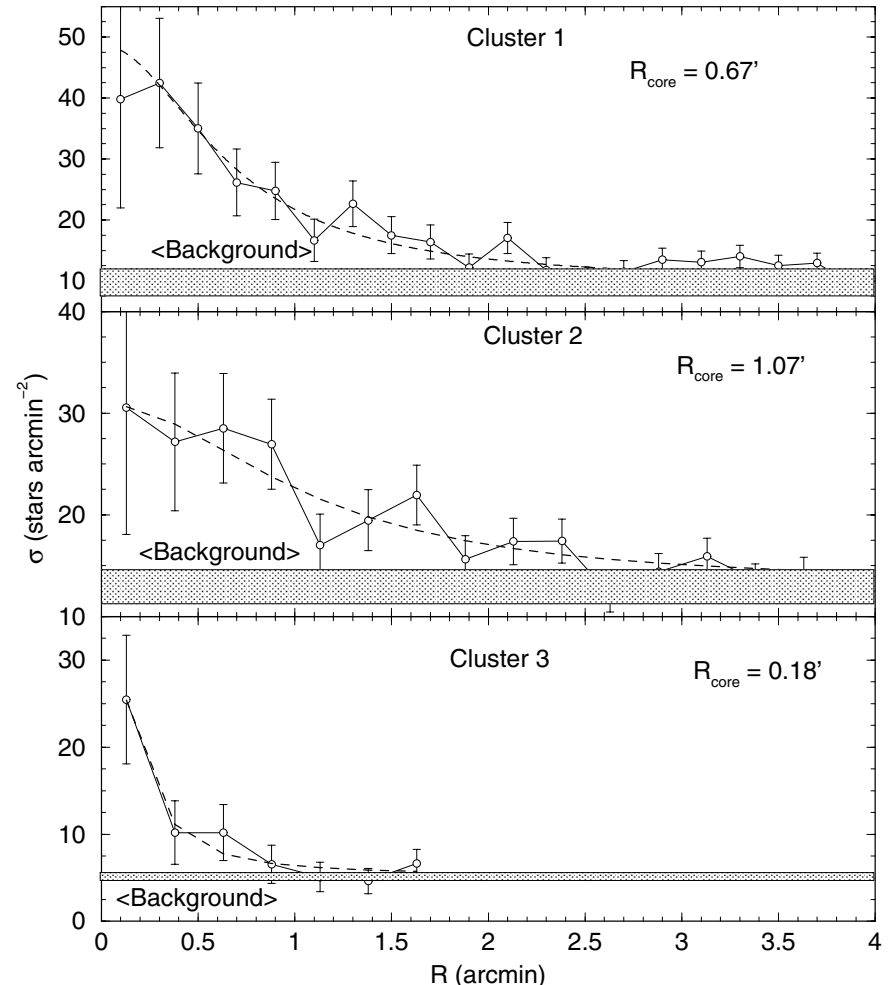

Fig. 5. Radial distribution of surface star density. The average background levels are shown as shaded rectangles; Poissonic errors are also shown. For Clusters 1 and 2 , magnitude $(J<15.5)$ and colour cutoffs have been applied to the object and offset fields. The dashed lines show a surface density profile fit to the radial distribution of stars; the resulting core radius for each cluster is indicated.

reaching a limiting radius of $R_{\lim } \approx 2.7^{\prime}$. The same is true for Cluster 2 (middle panel), although its profile is not as smooth as that of Cluster 1 . Cluster 2 extends to $R_{\lim } \approx 3.5^{\prime}$. The well-defined and smooth profile of Cluster 3 (bottom panel) is more concentrated than those of Clusters 1 and 2, extending to $R_{\lim } \approx 1.6^{\prime}$.

Although the clusters' spatial geometry may not be perfectly spherical, we still can apply King's law (1966) in order to derive first order structural parameters. A cluster core radius $R_{\text {core }}$ can be calculated by fitting a King surface density profile $\sigma(R)=\frac{\sigma_{0}}{1+\left(R / R_{\text {core }}\right)^{2}}$ to the background-subtracted radial distribution of stars. The resulting fits are also shown in Fig. 5, as dashed lines. Clusters 1 and 3 follow a King profile within the uncertainties, with $R_{\text {core }}=0.67^{\prime}$ and $R_{\text {core }}=0.18^{\prime}$, respectively. The radial density profile of Cluster 2 is not as smooth as those of Clusters 1 and 3, consequently, the King profile fit is not as good and the resulting $R_{\text {core }} \sim 1.07^{\prime}$ has to be taken as an estimate only. Using the cluster distances derived in Sect. 5 below, the linear core radii turn out to be $R_{\text {core }}=0.32 \pm$ $0.03 \mathrm{pc}, \sim 0.68 \pm 0.06 \mathrm{pc}$ and $0.21 \pm 0.02 \mathrm{pc}$, respectively, for Clusters 1, 2 and 3. Finally, the angular diameters of 5.4', 7.0' and $3.2^{\prime}$ (Table 1), convert to linear limiting diameters of $2.6 \pm$ $0.3 \mathrm{pc}, 4.4 \pm 0.4 \mathrm{pc}$ and $3.8 \pm 0.3 \mathrm{pc}$. Cluster 1 is very young (Sect. 5), indeed its diameter is comparable to typical values observed in infrared embedded clusters (Bica et al. 2003). The older Clusters, 3 and especially 2, have larger diameters which

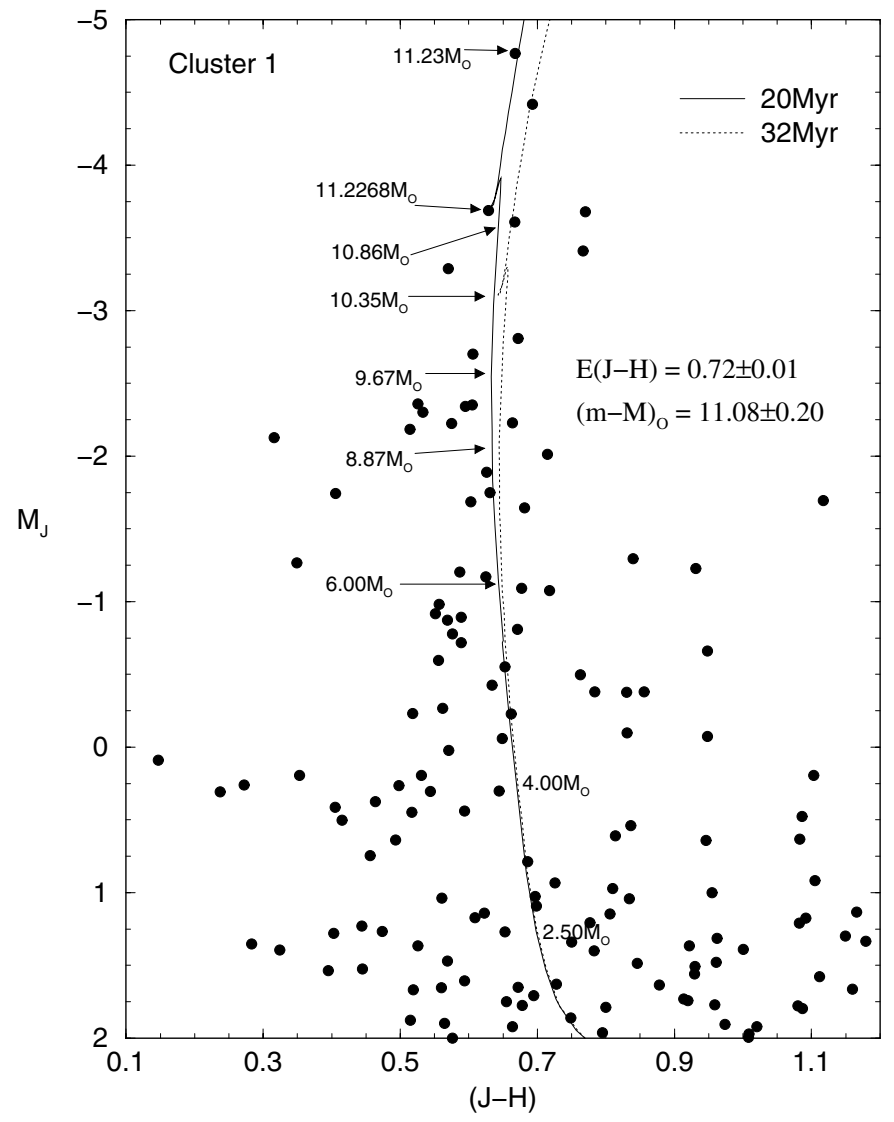

Fig. 6. Isochrone fitting to the $M_{\mathrm{J}} \times(J-H)$ CMD of Cluster 1 . Equally good fits are obtained for 20 and $32 \mathrm{Myr}$ isochrones, resulting in $E(B-V)=2.18 \pm 0.03$ and $d_{\odot}=1.64 \pm 0.19 \mathrm{kpc}$. Stellar masses along the main-sequence are indicated.

must be reflecting the stochastic effects of the Galactic tidal processes (Bonatto \& Bica 2003). In the case of the much older open cluster M 67, located about $1 \mathrm{kpc}$ outside the Solar circle, the limiting diameter is $\approx 12 \mathrm{pc}$, while Cluster 2 with a limiting diameter of $\approx 4.4 \mathrm{pc}$, is located $\approx 2 \mathrm{kpc}$ inside the Solar circle.

\section{Fundamental parameters}

In the following two sections we will base our analyses on stars extracted within $R_{\mathrm{CMD}}$ (Table 1 ). In order to derive cluster parameters we use solar metallicity Padova isochrones from Girardi et al. (2002) computed with the 2MASS $J, H$ and $K_{\mathrm{S}}$ filters (available at http://pleiadi.pd.astro.it/ lgirardi/-isoc_photsys.00/). The 2MASS transmission filters produced isochrones very similar to the Johnson ones, with differences of at most 0.01 in $(J-H)$ (Bonatto et al. 2004). For reddening and absorption transformations we use $R_{\mathrm{V}}=3.2$, and the relations $A_{J}=0.276 A_{\mathrm{V}}$ and $E(J-H)=$ $0.33 E(B-V)$, following Dutra et al. (2002) and references therein.

We show in Fig. 6 the isochrone fitting to the $M_{\mathrm{J}} \times(J-H)$ CMD of Cluster 1 . The $M_{\mathrm{J}}$ values are obtained after applying the distance modulus derived below for each cluster. The observed scatter in colour of stars along the MS makes it possible to fit it equally well with the 20 and 32 Myr isochrones, thus 


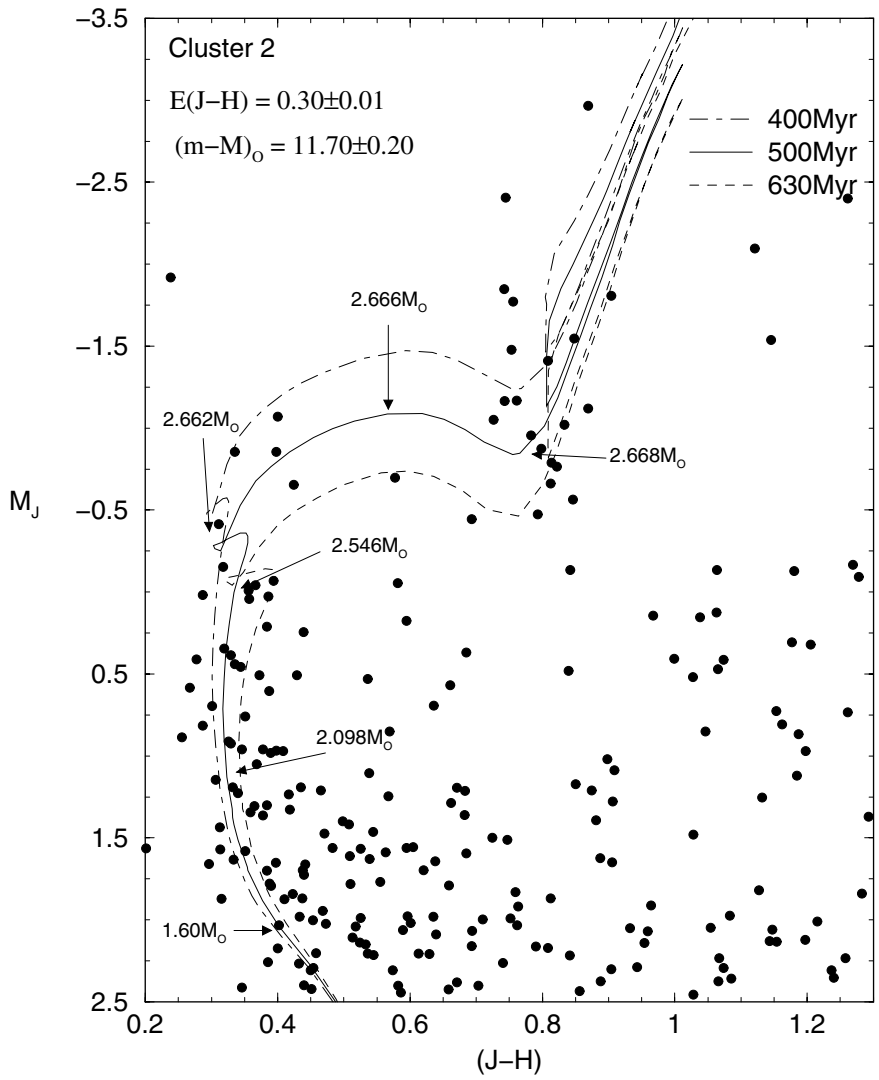

Fig. 7. Same as Fig. 6 for Cluster 2. Acceptable fits are obtained for 400-630 Myr isochrones, resulting in $E(B-V)=0.91 \pm 0.03$ and $d_{\odot}=2.19 \pm 0.21 \mathrm{kpc}$.

constraining the age to this narrow range. The fit and related uncertainties give a distance modulus $(m-M)_{0}=11.08 \pm$ $0.20, E(B-V)=2.18 \pm 0.03$ and $d_{\odot}=1.64 \pm 0.19 \mathrm{kpc}$. Representative stellar masses are indicated along the $20 \mathrm{Myr}$ isochrone.

The presence of giants in the CMD of Cluster 2 indicates an older age for this object. Indeed, allowing for the star scatter, the distribution of stars can be fitted by isochrones with ages in the range 400-630 Myr, as can be seen in Fig. 7. For Cluster 2 we derive $(m-M)_{0}=11.70 \pm 0.20, E(B-V)=0.91 \pm 0.03$ and $d_{\odot}=2.19 \pm 0.21 \mathrm{kpc}$.

The best fit for Cluster 3 is obtained with the $100 \mathrm{Myr}$ isochrone; however, this solution is strongly based on the membership assumption of a single evolved star. Arguing in favour of membership, the coordinates of that bright star $\alpha(2000)=07^{\mathrm{h}} 01^{\mathrm{m}} 01.10^{\mathrm{s}}$ and $\delta(2000)=-22^{\circ} 01^{\prime} 29.57^{\prime \prime}-$ place it at $0.18^{\prime}$ from the cluster's central position. Another solution, discarding the bright star, is obtained with the $32 \mathrm{Myr}$ isochrone. Both solutions are shown in Fig. 8. In any case, proper-motion information is necessary to derive the precise age for this cluster. For Cluster 3 we derive $(m-M)_{0}=12.97 \pm$ $0.20, E(B-V)=0.94 \pm 0.03$ and $d_{\odot}=3.93 \pm 0.35 \mathrm{kpc}$.

The $E(B-V)_{\text {FIR }}$ reddening values in Table 1 are overestimates, since they represent the dust column contribution integrated along the whole line of sight (up to the disk edge). Thus, using the exponential dust distribution model of Chen et al. (1999) and the distances derived from the CMDs, we

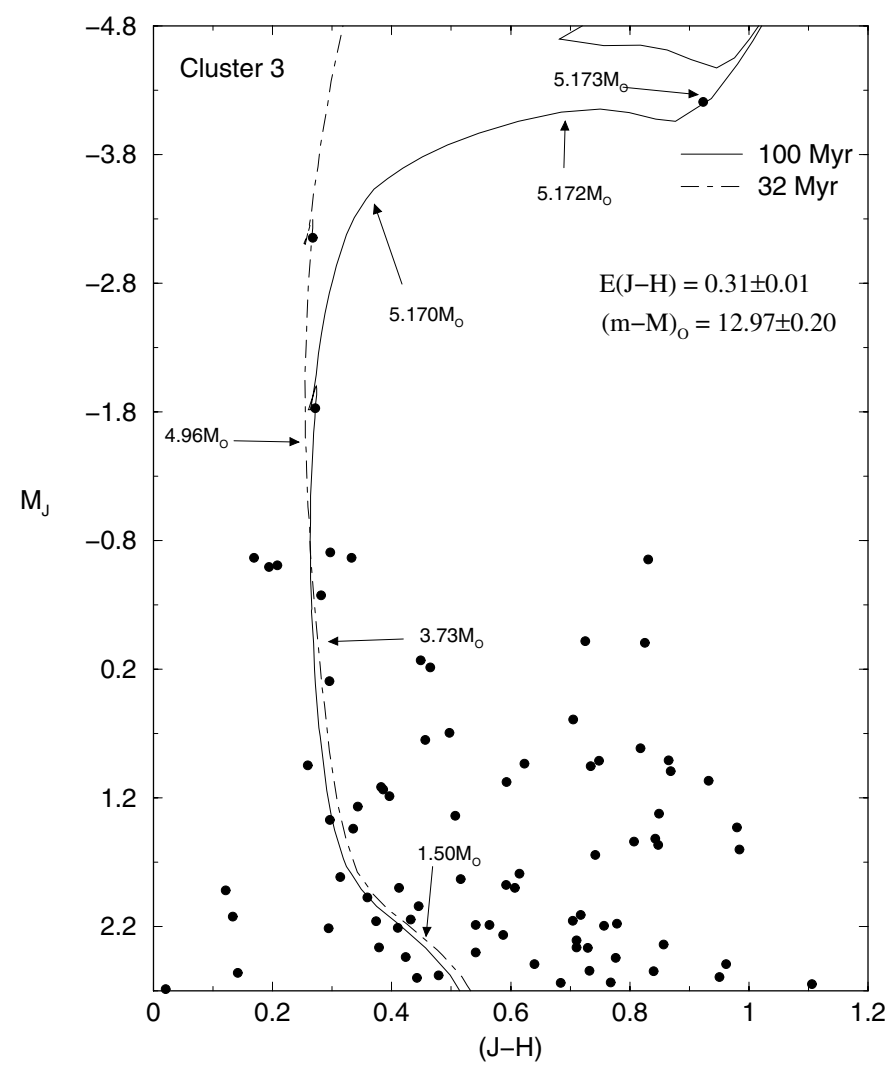

Fig. 8. Same as Fig. 6 for Cluster 3. Depending on the membership of a single evolved star, acceptable fits are obtained for 100 and $32 \mathrm{Myr}$ isochrones, resulting in $E(B-V)=0.94 \pm 0.03$ and $d_{\odot}=3.93 \pm$ $0.35 \mathrm{kpc}$.

obtain the following foreground reddening values for Clusters 1, 2 and 3, respectively: $E(B-V)=0.92,1.18$ and 1.17. The results for Clusters 2 and 3 are close to the reddening values derived from the CMDs, suggesting that for the directions to these clusters an exponential dust distribution law is a good approximation. Cluster 1 lies at a very low latitude, and as expected the agreement between reddening estimates is not good.

Cluster 1, at $1.6 \mathrm{kpc}$, does not appear to be related to the H II region complex Sh2-53, since this object is estimated to be at a kinematic distance of $\approx 4.3 \mathrm{kpc}$ (Georgelin \& Georgelin 1976). Cluster 1 seems to be related to the Sgr-Car arm, while Sh2-53 is probably related to the Sct-Cru arm. Cluster 3, at $3.9 \mathrm{kpc}$, appears to be located in the background of the closely projected young cluster which is at an estimated distance of $2 \mathrm{kpc}$ (Vogt \& Moffat 1972), as well as in the background of the $\mathrm{H}$ II region Brand 16 or RCW 14, located at $1.9 \mathrm{kpc}$ from the Sun (Brand \& Blitz 1993). The latter nebulae are probably related to the open cluster NGC 2367.

The fact that Cluster 3 is projected behind the nebula Brand 16 poses the question whether the cluster might be very young and physically related to it and to RCW 14. To address this question we plotted in a $(J-H) \times(H-K)$ diagram the stars inside $R_{\mathrm{CMD}}$ and the corresponding offset field extractions. This colour-colour diagram is sensitive to reddening and the presence of excess emission in the $K$ band related to protostellar disks (Soares \& Bica 2002, 2003, and references therein). 
We applied a cutoff of $J<15.5$ to avoid uncertainties larger than 0.08 in $J$. This procedure also avoids prohibitive errors in colours. As a result, we found no $(H-K)$ excess stars associated to Cluster 3. This result suggests that Cluster 3 is indeed in the background of Brand 16.

\section{Luminosity and mass functions}

Observed star counts as a function of magnitude (or mass) can be compared to theoretical predictions of number density as a function of luminosity (or mass). This can be used to test whether stars are present in numbers as expected and to estimate the cluster's total mass, taking into account stellar masses as low as $0.08 M_{\odot}$. This relation between number density and luminosity (or mass) is referred to as the LF (or MF) - Salpeter (1955).

The accurate determination of a cluster's LF (or MF) suffers from some problems, in particular (i) the contamination of cluster members by field stars; (ii) the observed incompleteness for low-luminosity (or low-mass) stars; and (iii) the mass segregation, which may affect even poorly populated, relatively young clusters (Scalo 1998). The 2MASS uniform sky coverage allows one to overcome, at least in part, points (i) - since suitable offset fields can be selected around the cluster and (iii) the entire cluster area can be included in the analyses. Thus, advanced stages of mass segregation would affect more significantly the analysis of very old, dynamically evolved clusters (e.g. M 67, Bonatto \& Bica 2003). This is not the case for the three new clusters dealt with in the present work.

Figure 9 depicts the LFs $\left(\phi\left(M_{\mathrm{J}}\right)\right)$ in the $J$ filter (shaded area) for the three new clusters, built as the difference of the number of stars in a given magnitude bin between object (continuous line) and average offset field (dotted line). The LFs are given in terms of the absolute magnitude $M_{\mathrm{J}}$, after applying the distance modulus derived in Sect. 5 for each cluster.

We recall that the LFs for Clusters 1 and 2 (Fig. 9) are built after applying magnitude $(J<15.5)$ and colour cutoffs to the objects and offset fields (Sect. 5).

The background-subtracted LFs of Clusters 1 and 2 present significant star excesses over the background, increasing up to $J \approx 14.2$ for Cluster 1 , and $J \approx 13.2$ for Cluster 2 . Due to the different distances of both clusters, the turnover in their LFs begins at $M_{\mathrm{J}} \approx 1.25$, corresponding to the spectral types A 2-A 3 . It should be noted that the bump at $-2.0 \leq M_{\mathrm{J}} \leq-0.5$ in the LF of Cluster 2 can be accounted for by the overlap in $M_{\mathrm{J}}$ of MS stars near the turnoff at $0.3 \leq(J-H) \leq 0.4$ with red giant stars at $0.7 \leq(J-H) \leq 0.9$ (Fig. 7). Despite the scarcity of stars in Cluster 3, this object still presents star excesses over the background which roughly increase towards the low-mass end (bottom panel), peaking at $J \approx 16.2\left(M_{\mathrm{J}} \approx 2.4, \mathrm{~F} 0\right)$. Spectral types and corresponding $M_{\mathrm{J}}$ for MS stars, taken from Binney \& Merrifield (1998), are displayed in the top panel of Fig. 9.

An estimate of the mass presently stored in stars in a cluster can be obtained by fitting the function $\phi(m)=\frac{\mathrm{d} N}{\mathrm{~d} m} \propto m^{-(1+\chi)}$ to the cluster's MF, restricted to the MS and turnoff. Then, the number of member stars is $N^{*}=\int_{m_{\text {low }}}^{m_{\text {high }}} \phi(m) \mathrm{d} m$, and the stellar mass is $M^{*}=\int_{m_{\text {low }}}^{m_{\text {ligh }}} m \phi(m) \mathrm{d} m$, where $m_{\text {low }}$ and $m_{\text {high }}$

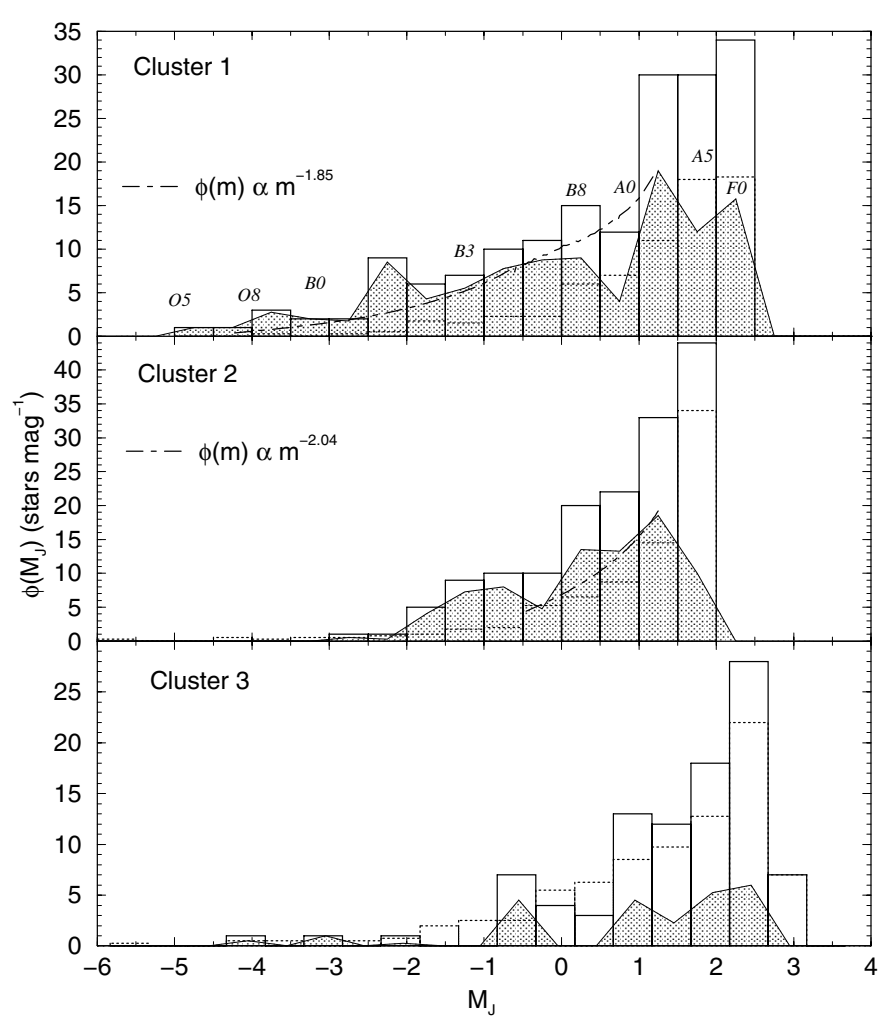

Fig. 9. Luminosity functions $\left(\phi\left(M_{\mathrm{J}}\right)\right)$ in terms of the absolute magnitude $M_{\mathrm{J}}$. For Clusters 1 and 2, magnitude $(J<15.5)$ and colour cutoffs have been applied to the object and offset fields. Continuous line: star counts in the cluster's area; dotted line: star counts in the offset fields; shaded area: background-subtracted LF. Representative MS spectral types, taken from Binney \& Merrifield (1998), are indicated in the top panel. MF fits $\left(\phi(m) \propto m^{-(1+\chi)}\right)$ are shown as dot-dashed lines.

define the range of stellar masses from the turnover at the low-MS up to the turnoff. The stellar mass-luminosity correspondence, necessary to convert the LFs in Fig. 9 into MFs, $\phi(m)=\phi\left(M_{\mathrm{J}}\right)\left|\frac{\mathrm{d} m}{\mathrm{~d} M_{\mathrm{J}}}\right|^{-1}$, has been obtained from the Padova isochrones which best fit the CMDs (Sect. 5): $20 \mathrm{Myr}$ for Cluster 1, 500 Myr for Cluster 2 and 100 Myr for Cluster 3.

Since Cluster 1 is young with no late-type stars observed in the CMD, we included in the fit the whole range of observed masses down to the turnover at $M_{\mathrm{J}} \approx 1.25$, which corresponds to a mass $m=2.51 M_{\odot}$. We point out that for high masses we are not dealing with OV stars (Fig. 6), in fact these very few stars are hot giants which do not significantly affect the analysis below. To estimate the mass of Cluster 2 we took into account separately the MS and evolved stars. We first fitted a MF to the MS stars from the turnover at $M_{\mathrm{J}}=1.25\left(m=2.037 M_{\odot}\right)$ up to $M_{\mathrm{J}}=-0.25\left(m=2.615 M_{\odot}\right)$ to avoid contamination by the overlapping giants, and then extrapolated this function to the turnoff $\left(M_{\mathrm{J}} \approx-0.50, m \approx 2.662 M_{\odot}\right)$. The mass of the giants $\left(M_{\mathrm{evol}}^{*}\right)$ has been estimated by counting the number of stars in each magnitude bin (after subtracting the MS stars in the overlap region) and multiplying this value by the average mass of the giants included in the bin. $M_{\mathrm{evol}}^{*}$ is given in Col. 4 of Table 2. The resulting MF fits for Clusters 1 and 2, transformed back to $\phi\left(M_{\mathrm{J}}\right)$, are shown as dot-dashed lines in Fig. 9 . The related parameters are given in Table 2. We also include in 
Table 2. Mass-function fit and related parameters.

\begin{tabular}{|c|c|c|c|c|c|c|c|c|c|c|}
\hline \multirow[b]{2}{*}{ Object } & \multicolumn{3}{|c|}{ Observed } & \multirow[b]{2}{*}{$\begin{array}{l}\text { Fit } \\
\left(M_{\mathrm{J}}\right)\end{array}$} & \multirow[b]{2}{*}{$\begin{array}{c}\chi \\
\left(m^{-(1+\chi)}\right)\end{array}$} & \multirow[b]{2}{*}{$\begin{array}{l}m_{\text {low }} \\
\left(M_{\odot}\right)\end{array}$} & \multirow[b]{2}{*}{$\begin{array}{l}m_{\text {high }} \\
\left(M_{\odot}\right)\end{array}$} & \multicolumn{3}{|c|}{ Calculated } \\
\hline & $\begin{array}{c}N_{\mathrm{MS}}^{*} \\
\text { (stars) }\end{array}$ & $\begin{array}{c}N_{\text {evol }}^{*} \\
\text { (stars) }\end{array}$ & $\begin{array}{l}M_{\text {evol }}^{*} \\
\left(M_{\odot}\right) \\
\end{array}$ & & & & & $\begin{array}{c}M^{*} \\
\left(M_{\odot}\right) \\
\end{array}$ & $\begin{array}{c}N_{\max }^{*} \\
\text { (stars) }\end{array}$ & $\begin{array}{l}M_{\max }^{*} \\
\left(M_{\odot}\right) \\
\end{array}$ \\
\hline Cluster 1 & 103 & 0 & 0 & $-4.75 \rightarrow 1.25$ & 0.85 & 2.515 & 11.22 & 147 & 755 & 382 \\
\hline Cluster 2 & 62 & 18 & 48 & $-0.25 \rightarrow 1.25$ & 1.04 & 2.037 & 2.662 & $89 \dagger$ & $2036 \dagger$ & $614 \dagger$ \\
\hline Cluster 3 & 25 & 1 & $\sim 5.2$ & - & - & - & - & $55 \dagger$ & - & - \\
\hline
\end{tabular}

Table notes. Column 5 is the $M_{\mathrm{J}}$ range to which the MF has been fitted. The values for Cluster 3 are derived from directly counting stars in each magnitude bin. $(\dagger)$ : includes MS and evolved stars. $N_{\max }^{*}$ and $M_{\max }^{*}$ are calculated by extrapolating the MF fits to $m_{\text {low }}=0.08 M_{\odot}$.

Table 2 the number of observed MS $\left(N_{\mathrm{MS}}^{*}\right)$ and evolved $\left(N_{\mathrm{evol}}^{*}\right)$ member stars, obtained by summing up the stars in each magnitude bin in the corresponding LFs.

The MF method underestimates both the mass and number of stars, since completeness effects affect the 2MASS observations in the low-mass end. More realistic values can be obtained by extrapolating the MFs derived above down to the theoretical stellar low-mass end $m_{\text {low }}=0.08 M_{\odot}$. The corresponding maximum number of stars $\left(N_{\max }^{*}\right)$ and mass $\left(M_{\max }^{*}\right)$ for Clusters 1 and 2 are given in the last two columns of Table 2, respectively.

Due to the small number of stars in the CMD, we could not apply the MF fit method to Cluster 3. Thus, we estimate the stellar mass in Cluster 3 by adopting an average mass per magnitude bin (based on the mass-luminosity correspondence taken from the 100 Myr Padova isochrone) and multiplying this value by the number of stars in each bin.

The MF slopes derived for Clusters 1 and 2 (Table 2) are comparable to, but somewhat flatter than, a standard Salpeter slope of $\chi=1.35$ (Binney \& Merrifield 1998). It is interesting to note that the extrapolated mass of Cluster 2 turns out to be larger than that of Cluster 1, in the opposite sense with respect to the observed mass. This fact can be accounted for by Cluster 2 having a steeper MF slope $(\chi=1.04)$ than Cluster 1 $(\chi=0.85)$.

None of the present objects is a massive open cluster (in the sense of say, $M^{*} \geq 10^{3} M_{\odot}$ ). Cluster 3 may be an evolutionary product of low-mass embedded clusters such as those described by Soares \& Bica $(2002,2003)$.

\section{Concluding remarks}

The recent discoveries of star clusters in the Galactic disk have shown that the census of these objects is not complete, even in the optical domain. In this paper we report the discovery of three optical open clusters in the Galaxy. Two clusters are in Scutum, at very low Galactic latitudes, and the third is in Canis Major. The present photometric and structural analyses make use mostly of $J$ and $H$ 2MASS All Sky data release photometry.

Arguments in favour of their open cluster nature are: (i) the visual contrast between the stellar concentrations in the cluster and background regions (Figs. 1-3); (ii) the CMDs which present well-defined sequences and are different from those for the offset field (Fig. 4); (iii) the radial star density profiles which show significant excesses over the background and can be fitted by a King law (Fig. 5); (iv) the constrained solutions of the isochrone fits to the CMDs, allowing a narrow range of ages (Figs. 6-8); and (v) the background-subtracted LFs which present significant star excesses over the background down to A 2-A 3 stars for Clusters 1 and 2, and to F 0 stars for Cluster 3 (Fig. 9). Completeness effects and photometric errors do not allow inferences on lower masses. For the sampled magnitude range, the number of member stars turns out to be 103, 80 and 26, respectively for Clusters 1, 2 and 3 .

Fundamental parameters for the objects have been derived by fitting solar-metallicity Padova isochrones to the 2MASS $M_{\mathrm{J}} \times(J-H)$ CMDs. Cluster 1 has an age $t=20-32 \mathrm{Myr}$, a reddening $E(B-V)=2.18 \pm 0.03$ and a distance to the Sun $d_{\odot}=1.64 \pm 0.19 \mathrm{kpc}$. For Cluster 2 we derive $t=$ 400-630 Myr, $E(B-V)=0.91 \pm 0.03$ and $d_{\odot}=2.19 \pm$ $0.21 \mathrm{kpc}$. For Cluster $3, t=32-100 \mathrm{Myr}, E(B-V)=0.94 \pm$ 0.03 and $d_{\odot}=3.93 \pm 0.35 \mathrm{kpc}$. At the above distances, the core radii for Clusters 1,2 and 3 turn out to be $0.32 \pm 0.03 \mathrm{pc}, 0.68 \pm$ $0.06 \mathrm{pc}$ and $0.21 \pm 0.02 \mathrm{pc}$, respectively. The linear limiting diameters are $2.6 \pm 0.3 \mathrm{pc}, 4.4 \pm 0.4 \mathrm{pc}$ and $3.8 \pm 0.3 \mathrm{pc}$, respectively, thus suggesting dynamical evolution effects for the older clusters.

For Clusters 1 and 2, a MF fit $\phi(m) \propto m^{-(1+\chi)}$ resulted in slopes $\chi=0.85$ and 1.04 , respectively. Thus, the observed stellar mass in Cluster 1 is $147 M_{\odot}$. In Cluster 2, including the evolved stars, it is $89 M_{\odot}$. The stellar mass in Cluster 3 is $\sim 55 M_{\odot}$. Extrapolating the MF fits down to the theoretical low-mass end $m_{\text {low }}=0.08 M_{\odot}$, the masses of Clusters 1 and 2 turn out to be $382 M_{\odot}$ and $614 M_{\odot}$, respectively.

The present study suggests that more optical open clusters are yet to be found in the Galaxy.

Acknowledgements. This publication makes use of data products from the Two Micron All Sky Survey, which is a joint project of the University of Massachusetts and the Infrared Processing and Analysis Center/California Institute of Technology, funded by the National Aeronautics and Space Administration and the National Science Foundation. We employed catalogues from CDS/Simbad (Strasbourg) and Digitized Sky Survey images from the Space Telescope Science Institute (US Government grant NAG W-2166) obtained using the extraction tool from CADC (Canada). We also made use of the WEBDA open cluster database. We acknowledge support from the Brazilian Institution CNPq. 


\section{References}

Alter, G., Ruprecht, J., \& Vanysek, V. 1970, in Catalogue of star clusters and associations + supplements, 2nd Edition (Budapest: Akad. Kiado)

Bica, E., Dutra, C. M., Soares, J., \& Barbuy, B. 2003, A\&A, 404, 223

Bica, E., Bonatto, C. J., \& Dutra, C. M. 2003, A\&A, 405, 991

Binney, J., \& Merrifield, M. 1998, in Galactic Astronomy (Princeton, NJ : Princeton University Press), Princeton series in astrophysics, QB857.B522

Bonatto, C., Bica, E., \& Girardi, L. 2004, A\&A, 415, 571

Bonatto, C., \& Bica, E. 2003, A\&A, 405, 525

Brand, J., \& Blitz, L. 1993, A\&A, 275, 67

Brand, J., Blitz, L., \& Wouterloot, J. G. A. 1986, A\&AS, 65, 537

Chen, B., Figueras, F., Torra, J., et al. 1999, A\&A, 352, 459

Chen, L., Hou, J. L., \& Wang, J. J. 2003, AJ, 125, 1397

Dias, W. S., Alessi, B. S., Moitinho, A., \& Lépine, J. R. D. 2002, A\&A, 389, 871

Dutra, C. M., Santiago, B. X., \& Bica, E. 2002, A\&A, 381, 219

Dutra, C. M., Bica, E., Soares, J., \& Barbuy, B. 2003, A\&A, 400, 533

Dutra, C. M., Santiago, B. X., Bica, E. L. D., \& Barbuy, B. 2003, MNRAS, 338, 253

Georgelin, Y. M., \& Georgelin, Y. P. 1976, A\&A, 49, 57

Girardi, L., Bertelli, G., Bressan, A., et al. 2002, A\&A, 391, 195

Janes, K., \& Adler, D. 1982, ApJS, 49, 425

King, I. 1966, AJ, 71, 64
Lyngå, G. 1987, in Computer based catalogue of open cluster data, 5th ed. (Strasbourg: CDS)

Mermilliod, J. C. 1996, in The origins, evolution, and destinies of binary stars in clusters, ASP Conf. Ser., 90, 475

Pfleiderer, J., Weinberger, R., \& Mross, R. 1977, in Star Cluster Symp. Budapest (Publ. Eøtvos Univ.), 5, 39

Rodgers, A. W., Campbell, C. T., \& Whiteoak, J. B. 1960, MNRAS, 121, 103

Salpeter, E. E. 1955, ApJ, 121, 161

Saurer, W., Seeberger, R., Weinberger, R., \& Ziener, R. 1994, AJ, 107, 2101

Scalo, J. 1998, in The Stellar Initial Mass Function, ed. G. Gilmore, \& D. Howell (San Francisco: ASP), ASP Conf. Ser., 142, 201

Schlegel, D. J., Finkbeiner, D. P., \& Davis, M. 1998, ApJ, 500, 525

Sharpless, S. 1959, ApJS, 4, 257

Skrutskie, M., Schneider, S. E., Stiening, R., et al. 1997, in The Impact of Large Scale Near-IR Sky Surveys, ed. Garzon et al. (Netherlands: Kluwer), 210, 187

Soares, J. B., \& Bica, E. 2002, A\&A, 388, 172

Soares, J. B., \& Bica, E. 2003, A\&A, 404, 217

Turner, D. G., Leonard, P. J. T., \& Madore, B. F. 1986, JRASC, 80, 166

Twarog, B. A., Ashman, K. M., \& Anthony-Twarog, B. J. 1997, AJ, 114,2556

Vogt, N., \& Moffat, A. F. J. 1972, A\&AS, 7, 133 